\title{
ORT.I 9 - Comparative Study of Vero Cell Growth and Virus Infection in Two Different Serum-Free Medium (SFM)
}

Kelly Araujo Lucio ${ }^{1 *}$; Viviane Silva Gomes Rodrigues'; Guilherme de Jesus da Silva ${ }^{1}$; Ygara da Silva Mendes ${ }^{1}$; Sheila Maria Barbosa de Lima ${ }^{1}$.

1Fiocruz/Bio-Manguinhos.

Introduction: Vero cell line, derived from African green monkey kidney, has been widely used for viral vaccine productions (WHO, 1998). To establish cell cultures in vitro, fetal bovine serum (FBS) is of importance for promoting cell growth because it contains growth factors, attachment and proliferation. However, using serum in cell cultures has a number of problems, particularly the contamination with adventitious agents (mycoplasma, viruses and prions). Moreover, it can make a serious problem in the downstream processing of recombinant proteins and vaccine production. The constant demand imposed by the regulatory and health agencies for greater quality, safety and consistency has motivated industries to develop culture media formulations without FBS. A major challenge currently faced by industry is to formulate consistent and robust media for anchorage-dependent cell lines used for vaccine production, such as Vero cells (Butler, 2013).

Objective: The aim of this work was to compare Vero cell growth and virus production in stationary cultures using two different serum-free medium VP-SFM and ProVERO-1.

Methodology: Vero cell stocks stored in liquid nitrogen, produced by Virological Technology Laboratory of Bio-Manguinhos (LATEV) was cultivated and infected with Chikungunya virus (CHIKV) in presence of the VP-SFM and ProVERO-1, both with serum-free environment. For comparison purposes, Vero cells were also cultivated in 199 media supplemented with FBS. Vero cells were grown until reaching a confluence of $95 \%$. CHIKV infection was carried with Multiplicity of infection (MOI) of 0.01 for $48 \mathrm{~h}$.

Results: The results showed that there was no difference in growth rate, but regarding morphology, Vero cells presented an elongated shape in VP-SFM, in comparison with a slightly branched characteristic in Pro Vero- 1 in the first $48 \mathrm{~h}$. Once confluent, they recovered their characteristic square shape, similar to cultivation in 199 medium. However, after $72 \mathrm{~h}$ of culture, VP-SFM medium presented a better performance and adaptation than ProVERO-1, probably due to the absence of epidermal growth factors in the latter. Cultures in VP-SFM medium showed similar performance to Vero cell cultures in 199 medium, although with different morphological characteristics in the first $24 \mathrm{~h}$. However, neither VP-SFM nor ProVERO- 1 reached the yields achieved by CHIKV production in 199 culture media at $48 \mathrm{~h}$ post infection.

Conclusion: The results showed that Vero cell lineage was able to grow in both culture media tested, although the VP-SFM medium showed better performance, regarding adaptation and viral production when compared with ProVERO-1. For the production of CHIKV in Vero cells, however, data suggest the need for optimization of the conditions, since the parameters used in this preliminary study were standardized for production in medium supplemented with FBS.

Keywords: cell Vero; VP-SFM and Pro VERO-1; Chikungunya virus 